\title{
Landmark studies in coronary heart disease epidemiology. The Framingham Heart Study after 70 years and the Seven Countries Study after 60 years
}

\author{
Barbara Cybulska, Longina Kłosiewicz-Latoszek \\ Institute of Food and Nutrition, Warsaw, Poland
}

\begin{abstract}
A bstract
This year we celebrate anniversaries of two prospective studies that have contributed most to our understanding of the epidemiology of coronary heart disease (CHD): the Framingham Heart Study (FHS) and the Seven Countries Study (SCS). The FHS was initiated 70 years ago and is continued in the subsequent generations using new research opportunities, including evaluation of the risk factors for chronic non-cardiovascular diseases. The SCS is now finished because the original study population are mostly deceased, and the study did not continue in the children and grandchildren of the participants. The FHS allowed identification of factors predisposing to CHD, which were referred to as "risk factors" for the first time. Based on the FHS findings, a multivariate model of the 10-year CHD risk was developed, known as the Framingham Heart Score. In addition, criteria of heart failure and risk factors for atrial fibrillation were defined. The SCS provided the first evidence for an association between nutrition and CHD and laid the foundations for recommending the Mediterranean diet for cardiovascular disease prevention.
\end{abstract}

Key words: Framingham Heart Study, Seven Countries Study, history, achievements

Kardiol Pol 2019; 77, 2: 173-180

\section{INTRODUCTION}

Two landmark studies for preventive cardiology, the Framingham Heart Study (FHS) and the Seven Countries Study (SCS), have their seventieth [1-3] and sixtieth [4] anniversaries this year, respectively. The FHS is currently in its third generation, while the SCS is finished because the original cohort in whom the study was initiated in 1958 are nearly all deceased by now. Some final publications from this study after 50 years of follow-up have recently appeared [5-8]. Thus, it is a good time now to summarise the results of both studies.

\section{THE HISTORY OF THE FRAMINGHAM HEART STUDY}

The FHS is the longest prospective observational study in which the population of the American town of Framingham, Massachusetts, was followed up. The aim of the study was, and continues to be, the identification of clinical and laboratory risk factors for cardiovascular disease (CVD), and currently other chronic diseases as well.
The history of the FHS has been recently recalled by its researchers $[9,10]$. The whole story began with President F.D. Roosevelt suffering from severe hypertension, which, left untreated, ultimately led to his death due to haemorrhagic stroke in April 1945. At that time, his blood pressure was 300/190 mmHg. Roosevelt's successor in the presidential office, Harry Truman, signed the National Heart Act in June 1948. In this act, the United States (US) Congress acknowledged that the health of the nation was at a significant risk from CVDs, including hypertension, and these diseases were the major cause of mortality in the US, accounting for one in three deaths. A sum of 500,000 US dollars was assigned to a 20-year epidemiological study of "heart disease," and the National Heart Institute was established, with its name changed to the National Heart, Lung, and Blood Institute in 1976. The FHS was run by this institution in partnership with the Boston University.

In 1947, while the National Heart Act was still in preparation, the US National Health Service appointed a young

Address for correspondence:

Prof. Barbara Cybulska, Institute of Food and Nutrition, ul. Powsińska 61/63, 02-903 Warszawa, Poland, tel: +48 225509681 , e-mail: barbara.cybulska@wp.pl Received: 15.10.2018 Accepted: 29.01.2019 Available as AoP: 29.01.2019 
Table 1. Some characteristics of all cohort studies $[11,14]$ of the Framingham Heart Study in modification

\begin{tabular}{|c|c|c|c|c|c|c|c|}
\hline \multirow[t]{2}{*}{ Cohort } & \multirow{2}{*}{$\begin{array}{l}\text { Total } \\
\text { (n) }\end{array}$} & \multirow{2}{*}{$\begin{array}{c}\text { Year } \\
\text { recruited }\end{array}$} & \multirow{2}{*}{$\begin{array}{c}\text { Age } \\
\text { [years] }\end{array}$} & \multicolumn{4}{|c|}{ Follow-up } \\
\hline & & & & $\begin{array}{l}\text { Examinations } \\
\text { (n)* }\end{array}$ & $\begin{array}{l}\text { Intervals } \\
\text { [years] }\end{array}$ & $\begin{array}{c}\text { Duration } \\
\text { [years] }\end{array}$ & $\begin{array}{c}\text { Sample size at last } \\
\text { examination (n) }\end{array}$ \\
\hline Original & 5209 & 1948-1953 & $28-74$ & 32 & 2 & 65 & 40 \\
\hline Offspring (and spouses) & 5124 & $1971-1975$ & $5-75$ & 9 & $4-8$ & 43 & 2430 \\
\hline Third generation & 4095 & 2002-2005 & $19-72$ & 2 & 6 & $\approx 10$ & 3411 \\
\hline Offspring spouses & 103 & $2003-2005$ & $47-85$ & 2 & 6 & $\approx 10$ & 68 \\
\hline Omni 1 & 507 & 1994-1998 & $27-78$ & 4 & $4-8$ & $\approx 15-20$ & 301 \\
\hline Omni 2 & 410 & 2003-2005 & $20-80$ & 2 & 6 & $\approx 10$ & 321 \\
\hline
\end{tabular}

*2015 - most recent year of the examination cycle

physician and officer, Dr. Meadors, to set up a proposal for a future epidemiological study. Dr. Meadors suggested studying the epidemiology of coronary heart disease (CHD) in a "normal" population, identifying factors that predispose to the development of the disease using clinical and laboratory tests, and undertaking a long-term follow-up. When the FHS was conceived, Dr. Meadors was appointed its first director.

The town of Framingham, Massachusetts, was chosen for several reasons. The Framingham Tuberculosis Demonstration Study was successfully conducted at that location from 1918 for six years [2]. The proximity of a large university medical centre in Boston allowed cooperation with cardiologists, and local physicians were keen to participate in the study.

On October 12, 1947, the US Public Health Service began supporting the study in cooperation with the Massachusetts State Department of Health and the Harvard Medical School, which was given the responsibility for professional and technical management of the study. Finally, Framingham was approved as the study location in November 1947.

\section{Study design}

The first study participant was examined on October 11, 1948 [1]. In 1948-1952, the original cohort of 5209 healthy volunteers aged 28-62 years was recruited [11]. Half of the participants were women. Family and medical history as well as anthropometric measurements were collected. The participants underwent physical examination and 12-lead electrocardiography (ECG) was performed. Blood and urine samples were collected for laboratory testing, and during subsequent decades the participants underwent periodic evaluation of selected clinical and laboratory parameters that might be associated with cardiac diseases, mostly due to atherosclerosis, with CHD being the primary target. In future years, five additional cohorts were created [9, 11, 12].

Analyses of standard 12-lead ECGs, performed at every FHS follow-up visit, contributed to the detection of CHD and development of epidemiology of left ventricular hyper- trophy, and allowed an early analysis of atrial fibrillation (AF) [13].

In 1971, a cohort of 5124 sons and daughters of the original participants (Framingham Offspring Study) was included in the study, and in 2002, the Framingham Third-Generation Study began, including 4095 participants aged 20 years or more who had at least one parent participating in the Framingham Offspring Study. The aim of the Framingham Offspring Study was to investigate the role of heritability of CHD and other CVDs, as well as related factors, while the objective of the Third-Generation Study was to explore genetic contribution to CVD.

In addition to those cohorts spanning three generations, three additional cohorts were recruited, including: 1) in 1994, the Omni 1 study, which included 506 participants from other ethnic groups than the original cohort; 2) in 2003, the New Offspring Spouse study, which included 103 spouses of the participants of the Framingham Offspring Study who had at least two children participating in the Framingham Third-Generation Study; and 3) in 2003, the Omni 2 study, which included 410 multi-ethnic participants, some being family members of the families participating in the Omni 1 study. Some characteristics of all the FHS cohort studies are presented in Table 1 [11, 14].

The initially evaluated risk factors for CHD in FHS were total cholesterol, blood pressure, smoking, and obesity. With time, as new diagnostic technologies and laboratory tests became available, FHS researchers introduced them into the protocols not only for evaluation of other coronary risk factors but also risk factors for other cardiac diseases. They included echocardiography, carotid artery ultrasound, magnetic resonance imaging of the heart and brain, computed tomography of the heart and great vessels, and Holter monitoring [12]. Apart from those measures, the FHS researchers expanded the study to identify genetic factors associated with CHD and other heart diseases. For this reason, the FHS was part of several large-scale international projects [11]. The analysis 
Table 2. Key dates in the history of the Framingham Heart Study [9-12]

\begin{tabular}{|c|c|}
\hline Year & Achievement \\
\hline 1948 & Beginning of the Framingham Heart Study \\
\hline 1957 & High blood pressure and cholesterol level increase the risk of CHD \\
\hline 1961 & Introduction of the term "risk factor" \\
\hline 1962 & Cigarette smoking associated with CHD \\
\hline 1964 & First report on stroke \\
\hline 1967 & Obesity and physical activity associated with the risk of $\mathrm{CHD}$ \\
\hline 1970 & High blood pressure increases stroke risk \\
\hline 1971 & Beginning of the Framingham Offspring Study \\
\hline 1971 & Criteria of heart failure \\
\hline 1974 & Diabetes associated with cardiovascular disease \\
\hline 1977 & Triglycerides, LDL-C, and HDL-C associated with the risk of CAD \\
\hline 1978 & AF associated with the risk of stroke \\
\hline 1988 & An inverse relationship between $\mathrm{HDL}-\mathrm{C}$ level and mortality \\
\hline 1988 & Isolated systolic hypertension directly associated with CHD \\
\hline 1994 & Description of the risk factors for $\mathrm{AF}$ \\
\hline 1994 & Beginning of the Omni 1 Framingham Heart Study — inclusion of participants from other ethnic groups \\
\hline 1996 & Description of progression from hypertension to heart failure \\
\hline 1998 & Presentation of a model for the estimated 10-year risk of CHD based on the risk factor profile (Framingham Risk Score) \\
\hline 2002 & Beginning of the Framingham Third Generation Study \\
\hline 2003 & $\begin{array}{l}\text { Beginning of the Omni } 2 \text { Framingham Heart Study - recruitment of multi-ethnic participants, in part family members } \\
\text { of Omni } 1 \text { study participants }\end{array}$ \\
\hline 2006 & $\begin{array}{l}\text { Launch of the single nucleotide polymorphism Health Association Resource - initiation of genetic studies of all } \\
\text { Framingham Heart Study cohorts using stored blood samples }\end{array}$ \\
\hline 2009-2010 & $\begin{array}{l}\text { Identification of hundreds of new genes associated with } \mathrm{CHD} \text { and major risk factors for heart disease, including body mass } \\
\text { index, blood pressure, diabetes, and AF }\end{array}$ \\
\hline
\end{tabular}

AF — atrial fibrillation; CAD — coronary artery disease; CHD — coronary heart disease; HDL-C — high-density lipoprotein cholesterol; LDL-C — low-density lipoprotein cholesterol

was possible because DNA samples were collected from 9300 members of the original cohort, the Offspring cohort, and the Third-Generation cohort in the 1990s and 2000s [15].

\section{RESULTS}

Important dates in the history of the FHS, along with the most important discoveries in CVD epidemiology, are summarised in Table 2 [9-12].

The introduction of the term "risk factor" by Kannel in 1961 after the publication of the first reports from FHS [16, 17] cannot be overestimated [18]. He defined a risk factor as any qualitative or quantitative variable, the presence of which in patients is statistically significantly associated with an increased incidence of, or mortality due to, specific disease.

According to Kannel and Schatzkin [19], "a risk factor for $\mathrm{CHD}$ is a characteristic of a person (demographic, psychologic, anatomic, physiologic) that increases the likelihood (risk) of that person developing some manifestation of CVD. The risk factor is not only statistically associated with CVD incidence, but as a result of meeting several criteria, it is considered to be causally related to the disease. These criteria of causality include the following: Consistency of association. The association should be found consistently within different studies of a variety of populations by using different methods of investigation... Strength of association. Persons exposed to a risk factor should developed substantially more disease than those not exposed (cohort approach)... Biologic plausibility. The association should be explicable in terms of and compatible with the existing scientific knowledge in both basic and clinical sciences... Time sequence. The presence of the risk factor should precede the development of disease... Dose-response gradient. The strength of the association should increase with the duration or intensity of the exposure... Potential for intervention. Alteration of the risk factor, possibly in conjunction with changes in other risk factors, should alter the risk of developing disease."

Introduction of low-density lipoprotein cholesterol (LDL-C) and high-density lipoprotein cholesterol (HDL-C) 
level measurements and the discovery of a positive association between LDL-C level and CHD risk, and a negative association between HDL-C level and CHD risk were of major importance for CVD prevention. Elucidation of the role of LDL-C led to clinical trials with LDL-C level-reducing drugs, primarily statins [20]. Major FHS achievements also include developing a multivariate model of the 10-year risk of $\mathrm{CHD}$ in relation to gender, age, smoking, systolic blood pressure, total cholesterol level, and HDL-C level (Framingham Heart Study Model). This model was used in the National Cholesterol Education Programme Adult Treatment Panel III guidelines [21]. Recently, the Framingham risk model was replaced with another risk calculator designed for primary prevention, the Pooled Cohort Risk Assessment Equation, which estimates the 10-year risk of fatal and non-fatal myocardial infarction and fatal and non-fatal stroke [22]. The new risk calculator has been used in the 2013 American College of Cardiology/American Heart Association (ACC/AHA) Guideline on the Treatment of Blood Cholesterol to Reduce Atherosclerotic Cardiovascular Risk in Adults [23].

The achievements of the FHS are not limited to atherosclerotic CVD risk factors but also include identification of $\mathrm{AF}$ as a potent risk factor for stroke [9], which laid the foundations for controlled clinical trials on anticoagulant and antiarrhythmic drugs in patients with AF. Based on the results of these studies, anticoagulation became standard therapy in AF. It was also shown that hypertension is the major cause of heart failure at the population level, and the clinical criteria for heart failure were defined. Echocardiography showed that clinical signs and symptoms of heart failure may also develop in subjects with preserved left ventricular systolic function (normal left ventricular ejection fraction) at the time of diagnosis. Medications that reduce mortality, such as $\beta$-blockers and angiotensin-converting enzyme inhibitors, were introduced in the 1990s and led to improved survival among patients with heart failure.

The FHS is ongoing and currently focuses on the effect of genes, various proteins, and other substances present in the body on the development of a wide range of conditions, from heart disease and obesity to Parkinson's disease and dementia [3]. Thanks to genetic studies, among them those targeting mutations at specific nucleotides in the DNA molecule, it has been possible to identify rare variants of genes associated with CHD, diabetes and lipids, body mass index, and blood pressure [11, 12]. However, according to Elosua et al. (2009) [24], "the strength of association between genetic variants and the risk of CHD is small. Most of the genes would not initially have been classified as candidate gene, except for the LDL receptor gene and proprotein convertase subtilisin/kexin 9 gene, which were included in the list of candidate genes because of their relevance in lipid metabolism." In contrast to the above-mentioned opinion, nine years later, with increasing knowledge, Knowles and Ashley [25] wrote: "Despite early criticism, most recent genetic scores have demonstrated significant improvement in performance for risk prediction in cardiovascular disease." Genetic factors for CHD have been reviewed in recent articles [26, 27].

Some critics have indicated the lack of baseline study presentation and the mixing up data from the original population and successive cohorts, in relation to some parameters. In fact, the FHS has not been conducted in a single original cohort throughout all decades and in relation to the same factors. The investigators gradually extended the cohort by adding descendants and spouses of the original participants, included new factors to be validated, with regard to their association with heart disease, and evaluated new diagnostic procedures towards their usefulness in clinical practice. Thus, the populations and duration of follow up of different individual parameters varied. In this context the term "longitudinal" used in relation to the FHS as a whole study is not justified. A better definition seems to be the longest-running, multigenerational cohort study.

\section{THE HISTORY OF THE SEVEN COUNTRIES STUDY}

While the FHS was the first epidemiological study of CHD risk factors in a single population, the SCS was the first prospective epidemiological study of CHD at the international level, performed in population samples of different countries and cultures [8]. The main goal of the study was to assess whether differences in the CHD rate (if present) may be explained by dietary habits [4]. The focus on nutrition was based on previous observations that this factor may have an effect on the incidence of CHD. This hypothesis was advanced in the late 1940s by Ancel Keys, a physiologist from the University of Minnesota. As reported by Menotti and Puddu [8] in an article to commemorate the $50^{\text {th }}$ anniversary of the SCS, this hypothesis was based on the results of controlled experiments to evaluate the effect of intake of various fats on cholesterol level, and unsystematised observations made during travel - together with a renowned Boston cardiologist, Dr. Paul White - to many countries with low rates of cardiac disease and low cholesterol levels. A number of these observations were made in Mediterranean countries, which prompted initiation of a multicentre international study later known as the Seven Countries Study of Cardiovascular Diseases [5]. According to Menotti and Puddu [8], a fundamental step towards this study was the 1952 meeting of Keys with Prof. Bergami, a Naples university physiologist, who invited Keys to investigate local low cholesterol levels and the paucity of heart attacks, except for among more affluent people. In 1954-1957, Keys undertook pilot studies in populations of various countries (Finland, Greece, Japan, Spain, and South Africa), being actively supported by Dr. Paul White. These studies provided suggestive evidence that heart attack rates were high in the US, Finland, and among Europeans in South Africa but low in the Mediterranean 
countries. These preliminary studies were a prelude to the formal SCS, which was initiated in 1958 and preceded by a 1957 pilot study in the Italian town of Nicotera [4]. The headquarters of the SCS research team was based at the University of Minnesota in Minneapolis.

During the first years, the SCS was mostly supported by US Public Health Service grants along with the National Heart, Blood, and Lung Institute and the American Heart Association. Individual cohort studies were supported by national organisations (foundations, societies, institutes, and ministries).

\section{Study design}

The study enrolled 12,763 healthy men aged $40-59$ years from 16 cohorts in seven countries (one cohort from the USA, two from Finland, one from the Netherlands, three from Italy, two from Croatia, three from Serbia, two from Greece, and two from Japan) [4]. In consequence, the study involved populations that were culturally, agriculturally, and nutritionally diverse.

At the beginning of the study, detailed data were collected on nutrition over a week at various times of the year (based on food diaries) in randomly chosen subgroups of 20-50 men in all 16 cohorts. Overall, the nutrition sub-study included 498 men. In subgroups of 13 cohorts, duplicates of food products consumed during a week (3282 days overall) were collected, weighted, and sent to a central laboratory for chemical analysis of their composition. Thus, data were available both on food intake and composition of consumed foods [6].

Much attention was paid to questionnaire standardisation. Principal SCS investigators underwent training at the University of Minnesota. Blackburn et al. [28] developed an objective classification system for ECG. The criteria and codes became known around that time as the Minnesota Code.

Systematic monitoring of CHD and other cardiovascular events was undertaken during the first 10 years of follow-up in all cohorts. Data on CHD deaths and other causes of mortality were collected for at least 25 years in all cohorts, and for up to 50 years in 13 of 16 cohorts [5].

\section{RESULTS}

Recently, an analysis of the association between baseline intake of fatty acids, food products, and dietary score and the 50-year all-cause mortality of the cohorts has been published [6]. The lowest all-cause mortality rates were observed in the rural Crete cohort and among Belgrade university professors, and the highest in the rural East Finland and Slavonia cohorts. The difference between extreme cohorts with regard to the mean age at the time of death was 12 years. In the 1960s, the average intake of saturated fatty acids (SFAs) and trans isomers of fatty acids and solid fats was high in the northern European cohorts, while intake of mono- and polyunsaturated fatty acids and plant oils was high in the Mediterranean regions. Low overall fat intake was noted in Japan.
For the 50-year all-cause mortality, a significant positive correlation was found with energy intake, and negative correlations with the monounsaturated to saturated $(\mathrm{M} / \mathrm{S})$ fat intake ratio, and the monounsaturated + polyunsaturated to saturated + trans $(\mathrm{M}+\mathrm{P} / \mathrm{S}+\mathrm{T})$ fat intake ratio [6]. An analysis of intake of different food groups showed negative correlations between all-cause mortality and mean population intake of plant food (including plant oils), plant oils separately, and the ratio of plant oil to solid fat intake. Animal food consumption showed no association with all-cause mortality, but a diet characterised by a high Mediterranean Adequacy Index (MAI) [29], indicating a predominance of plant over animal food intake, was inversely associated with all-cause mortality. MAI values were high in the Mediterranean cohorts and Japan but very low in USA, Finland, and the Netherlands.

Recently, a study has also been published on the relations between saturated fat, sucrose, and food group intake, as well as the Mediterranean-like diet (evaluated by MAI) at baseline and the 50-year CHD mortality [7]. Mortality rates due to CHD showed a nearly sevenfold difference between the cohorts. Fifty-year CHD mortality of the cohorts was shown to be highly significantly correlated with the mean population intake of SFA, solid fats, sweet products, animal foods, and sucrose. In contrast, no association was shown with the intake of naturally occurring sugars. Intake of plant foods, starch, and MAI correlated inversely with coronary deaths.

These results confirmed the earlier SCS findings at 10, 15, 20 , and 25 years in relation to SFA intake [5]. Overall, high SFA intake, leading to high cholesterol levels in the population, favoured high CHD mortality. On the other hand, the SCS survey established that high ratios of polyunsaturated to saturated and monounsaturated to SFAs have a protective effect, as they correlated inversely with coronary deaths. Of note, a negative correlation was found between flavonoid intake and coronary deaths over 25 years.

Regarding consumed foods, the 25-year CHD mortality showed a positive association with the consumption of milk, butter, meat, sugar, and pastries, and was negatively associated with the consumption of plant oils, legumes, and alcohol. A positive correlation was found with animal food intake, and negative correlations with plant food intake and MAI [30].

These observations at 25 and 50 years of follow-up support the concept of the Mediterranean diet as a model diet for CHD prevention, inscribed by the United Nations Educational, Scientific, and Cultural Organisation (UNESCO) on the Representative List of the Intangible Cultural Heritage of Humanity.

After the death of Prof. Ancel Keys in 2004, criticism was voiced regarding the SCS and its discoveries, questioning the scientific merit of the study. A response to nearly all criticisms was included in a recently published white paper [31]. A summary of issues discussed in the white paper was presented in a recent article by Menotti and Puddu [8]. 
Below, we present these criticisms and responses by the proponents of SCS findings that have been included in these two papers.

The opponents of the SCS claim that SCS investigators selected the participating countries in the way that led to the desired results, that is, the finding of a significant association between dietary fat and mostly SFAs with $\mathrm{CHD}$. In response, the proponents of the SCS noted that the participating populations were probably chosen due to their contrasting nutritional patterns, to allow evaluation of whether these differences were associated with different $\mathrm{CHD}$ rates. Prior to the study, actual nutritional patterns and CHD rates were not known.

The investigators alleged that France was purposefully excluded from the SCS, as the French paradox might disturb the expected results. However, it is true that the French investigators declined to participate in the main study, although they took part in the pilot SCS study in Nicotera in 1957, and the French paradox was not known at that time.

Another criticism was related to the fact that data from Greece were collected during Lent. Indeed, a part of the nutritional study was purposefully undertaken during that period, and no significant differences were found in both Greek cohorts regarding the types and amount of foods and nutrients consumed compared to other periods of the year.

An objection was raised that the analysis did not include sugar as a dietary component possibly linked to CHD. Sugar was taken into account as a separate food item, and it was shown that its intake was directly associated with the 25-year mortality due to CHD from the ecological point of view, but to a lesser extent than food groups rich in saturated fat, and saturated fat considered separately.

Two figures erroneously attributed to the SCS have been contested. One of them appeared in a 1953 publication by Keys [32], and was also shown earlier during his presentation at a symposium at Mount Sinai Hospital, New York. It shows an association between fat calories, as a percentage of total calories in a diet (based on data from the Food and Agriculture Organization) and deaths due to heart disease (based on data from the World Health Organization) in six countries (Australia, Canada, England and Wales, Italy, Japan, and the USA). Keys chose these six countries because they could provide the most reliable data on nutrition and statistical data regarding mortality that were available at that time.

Thus, as the above-mentioned figure was shown more than a decade before the first SCS publication, it obviously cannot be derived from this study. Long before the SCS, based on the data available at that time, Keys suspected that atherosclerosis is not a simple consequence of aging, and nutrition may have an impact on the clinical sequelae of atherosclerosis. This suggested that $\mathrm{CHD}$ might be preventable.

The other figure appeared in an article published by two statisticians in 1957, also before the SCS was initiated [33], and reported data for 22 countries. Critics suggested that based on these data, no correlation could be seen between fat intake and deaths due to atherosclerosis-linked degenerative heart disease. However, a positive correlation is clearly visible, although it is weaker. According to Keys, the authors of this figure used data on nutrition and cardiac deaths regardless of their quality. Keys also noted that it is possible that data on nutrition in some countries presented on that figure did not reflect an association with $\mathrm{CHD}$ due to inclusion of a large proportion of immigrants, whose diet was affected by their culture and country of origin. In addition, Keys noted a temporal problem the authors had with that figure, because data on nutrition were mostly from 1954, and data on mortality were derived from earlier years (1950-1952). In the meantime, nutrition might have changed, and it is likely that earlier data on nutrition in a given country, between the mid and late 1940s, might show a stronger correlation with coronary deaths in the early 1950 s compared to a later nutrition pattern in the population (as reflected by data from 1954).

It should be stressed again that both figures were published before initiation of the SCS, and of the six countries that were reported on the first figure, only three were later included in the SCS. Similarly, of 22 countries that were reported on the second figure, only six were later included in the SCS.

The opponents of the SCS concluded that the strong association between fat intake and CHD shown in the SCS promoted substitution of carbohydrates for fat, which might have contributed to the obesity epidemic. It should be stressed, however, that the SCS never showed a strong correlation between overall fat intake and CHD, and it was never suggested to substitute carbohydrates for fat based on these study findings.

Another objection was that the SCS relied too much on the ecological associations between the cohorts, which are not necessarily valid aetiologically. In response to this objection, it was stated that in all reports due caution was executed when relating aetiological validity to ecological associations.

Menotti and Puddu [8] concluded that criticisms of the SCS are largely based on fake news, and the critics did not read the original monographies and publications, passing their biased interpretations of the results to various media.

The SCS showed a significant positive association between SFA intake and the mean cholesterol level, and between the mean cholesterol level and CHD incidence [4]. Thus, it confirmed that the risk of CHD was related to the cholesterol level, as shown in a single population in FHS [16].

In the context of cholesterol level being an established strong risk factor, interesting observations that need to be explained have been made in the Japanese population after the SCS [34]. As already mentioned, the study showed the lowest mortality due to $\mathrm{CHD}$ in the Japanese cohorts. This was attributed to very low cholesterol levels.

The authors of the above-mentioned post-SCS study noted that mortality trends in Japan, Australia, Canada, France, 
Spain, Sweden, and the United Kingdom showed continuing decrease in mortality due to CHD in all these countries in 1980-2007, which was accompanied by a decrease in cholesterol levels in all countries except for Japan, where cholesterol levels were found to increase. Thus, Japan was characterised by a unique reduction in coronary mortality despite increasing cholesterol levels. The direction and size of changes in other risk factors (reduction of blood pressure levels and smoking, and increase in body mass index and the prevalence of diabetes) were similar to those seen in other countries. The authors suggested that some other protective factors unique for Japan might have played a role. Among these, nutritional factors were suggested, including a high intake of long-chain polyunsaturated fatty acids (PUFA n-3).

It should be noted, however, that cholesterol levels in the male Japanese population in 2008, which are higher compared to 1980 , are similar to cholesterol levels in American men in 2008, which are reduced compared to 1980. Similar relations were found in Japanese women in comparison to Australian women.

\section{SUMMARY}

Both the FHS and the SCS have played a major role in elucidating the causes of atherosclerotic CVD. The major legacy of the FHS, an observational study performed in a single population, is the identification of risk factors for CHD and introduction of the term "risk factor" in cardiology. Based on FHS findings, a model for predicting the 10-year risk of $\mathrm{CHD}$, known as the Framingham Risk Score, was developed and introduced into clinical practice. The FHS also showed that high blood pressure and AF were risk factors for stroke, and it defined the criteria for heart failure and risk factors for AF. The discovery of risk factors for CVD is highly important. It should be noted that the FHS is ongoing and focuses on the identification of genetic markers of CVD risk, along with risk factors for other diseases. The FHS has inspired many epidemiological studies all over the world.

The SCS was the first multi-population observational study performed in 16 cohorts that evaluated the effect of nutrition on $\mathrm{CHD}$ risk. Its discoveries that nutritional differences have a significant effect on all-cause and CHD mortality are of vital importance. SCS showed that populations with low intake of saturated fatty acids and solid fats, high dietary $M / S$ and $\mathrm{M}+\mathrm{P} / \mathrm{S}+\mathrm{T}$ ratios, high intake of plant foods and plant oils, and a high ratio of plant to animal food consumption were at low risk. This pattern of nutrition was typical of the Mediterranean cohorts that participated in this study. Based on that discovery, the Mediterranean diet is now the gold standard in CVD prevention [35,36]. A very strong argument in favour of this diet is the fact that life expectancy in cohorts traditionally using the Mediterranean diet is on average 12 years longer.

Finally, it is worth to stress the basic difference between these two landmark studies. FHS is the longest-running pro- spective epidemiologic study of CHD in history conducted in multigenerational community-based cohorts, whereas SCS was the first prospective epidemiological study of CHD performed in several cohorts of different countries and cultures.

\section{Conflict of interest: none declared}

\section{References}

1. Meadors GF. Memorandum to Chief. Heart Disease Control Section. US Public Health Service. Framingham Heart Study archives. Nov. 1. 1948.

2. Dawber TR, Meadors GF, Moore FE. Epidemiological approaches to heart disease: the Framingham Study. Am J Public Health Nations Health. 1951; 41(3): 279-281, indexed in Pubmed: 14819398.

3. Levy B. Framingham at 70: celebrating a landmark heart study. 9 Feb. 2018. NIH Intramural Research Program.

4. Keys A, Blackburn H, Menotti A, et al. Coronary heart disease in seven countries. Circulation. 1970; 41(Suppl. 1): 1-211.

5. Menotti A, Puddu PE. How the seven countries study contributed to the definition and development of the mediterranean diet concept: a 50-year journey. Nutr Metab Cardiovasc Dis. 2015; 25(3): 245-252, doi: 10.1016/j.numecd.2014.12.001, indexed in Pubmed: 25650160

6. Menotti A, Kromhout D, Puddu PE, et al. Baseline fatty acids, food groups, a diet score and 50-year all-cause mortality rates. An ecological analysis of the Seven Countries Study. Ann Med. 2017; 49(8): 718-727, doi: 10.1080/07853890.2017.1372622, indexed in Pubmed: 28847158.

7. Kromhout D, Menotti A, Alberti-Fidanza A, et al. Comparative ecologic relationships of saturated fat, sucrose, food groups, and a Mediterranean food pattern score to 50-year coronary heart disease mortality rates among 16 cohorts of the Seven Countries Study. Eur J Clin Nutr. 2018; 72(8): 1103-1110, doi: 10.1038/s41430-018-0183-1, indexed in Pubmed: 29769748.

8. Menotti A, Puddu PE. Can we still learn from the Seven Countries Study? Curr Opin Lipidol. 2018; 29(4): 313-317, doi:10.1097/MOL.0000000000000520, indexed in Pubmed: 29708924.

9. O'Donnell C, Elosua R. Cardiovascular risk factors. Insights From Framingham Heart Study. Rev Esp Cardiol. 2008; 61(3): 299-310, doi:10.1016/s1885-5857(08)60118-8.

10. Mahmood SS, Levy D, Vasan RS, et al. The Framingham Heart Study and the epidemiology of cardiovascular disease: a historical perspective. Lancet. 2014; 383(9921): 999-1008, doi: 10.1016/S0140-6736(13)61752-3, indexed in Pubmed: 24084292.

11. Tsao CW, Vasan RS. Cohort Profile: The Framingham Heart Study (FHS): overview of milestones in cardiovascular epidemiology. Int JEpidemiol. 2015; 44(6): 1800-1813, doi: 10.1093/ije/dyv337, indexed in Pubmed: 26705418.

12. Hajar R. Framingham contribution to cardiovascular disease. Heart Views. 2016; 17(2): 78-81, doi: 10.4103/1995-705X.185130, indexed in Pubmed:27512540.

13. Magnani JW, Newton-Cheh C, O'Donnell CJ, et al. Development and application of a longitudinal electrocardiogram repository: the Framingham Heart Study. J Electrocardiol. 2012; 45(6): 673-676, doi: 10.1016/j.jelectrocard.2012.06.016, indexed in Pubmed: 22832152.

14. Urooj T. Framingham Heart Study: a review of research design. Interventions Obes Diabetes 2018; 1(1): IOD.000505.

15. Jaquish CE. The Framingham Heart Study, on its way to becoming the gold standard for Cardiovascular Genetic Epidemiology? BMC Med Genet. 2007; 8: 63, doi: 10.1186/1471-2350-8-63, indexed in Pubmed: 17916250. 
16. Dawber TR, Moore FE, Mann GV, et al. Coronary heart disease in the Framingham study. Am J Public Health Nations Health. 1957; 47(4 Pt 2): 4-24, indexed in Pubmed: 13411327.

17. Dawber TR, Kannel WB, Revotskie N, et al. Some factors associated with the development of coronary heart disease: six years' follow-up experience in the Framingham study. Am J Public Health Nations Health. 1959; 49: 1349-1356, indexed in Pubmed: 13814552.

18. Kannel WB, Dawber TR, Kagan A, et al. Factors of risk in the development of coronary heart disease: six year follow-up experience. The Framingham Study. Ann Intern Med. 1961; 55: 33-50, indexed in Pubmed: 13751193.

19. Kannel WB, Schatzkin A. Risk factor analysis. Prog Cardiovasc Dis. 1986; 26(4): 309-332, indexed in Pubmed: 6229811.

20. Fulcher J, O'Connel R, Voysey M, et al. Efficacy and safety of LDL-lowering therapy among men and women: meta-analysis of individual data from 174000 participants in 27 randomised trials. Lancet. 2015; 385(9976): 1397-1405, doi: 10.1016/s01406736(14)61368-4.

21. Third Report of the Expert Panel on Detection, Evaluation, and Treatment of High Blood Cholesterol in Adults (ATP III Final Report). Circulation. 2002; 106(25): 3143-3421.

22. Goff D, Lloyd-Jones D, Bennett G, et al. 2013 ACC/AHA Guideline on the Assessment of Cardiovascular Risk. J Am Coll Cardiol. 2014; 63(25): 2935-2959, doi: 10.1016/j.jacc.2013.11.005.

23. Stone NJ, Robinson JG, Lichtenstein AH, et al. 2013 ACC/AHA Guideline on the Treatment of Blood Cholesterol to Reduce Atherosclerotic Cardiovascular Risk in Adults. A Report of the American College of Cardiology/American Heart Association Task Force on Practice Guidelines. J Am Coll Cardiol. 2014; 63(25 Pt B): 2889-2934.

24. Elosua R, Lluis C, Lucas G. Research into the genetic component of heart disease: from linkage studies to genome-wide genotyping. Rev Esp Cardiol. 2009; 09(supl B): 24-38.

25. Knowles JW, Ashley EA. Cardiovascular disease: The rise of the genetic risk score. PLoS Med. 2018; 15(3): e1002546, doi: 10.1371/journal.pmed.1002546, indexed in Pubmed: 29601582.

26. Khera AV, Kathiresan S. Genetics of coronary artery disease: discovery, biology and clinical translation. Nat Rev Genet. 2017; 18(6): 331-344, doi: 10.1038/nrg.2016.160, indexed in Pubmed: 28286336.

27. Dogan MV, Grumbach IM, Michaelson JJ, et al. Integrated genetic and epigenetic prediction of coronary heart disease in the
Framingham Heart Study. PLoS One. 2018; 13(1): e0190549, doi: 10.1371/journal.pone.0190549, indexed in Pubmed: 29293675.

28. Blackburn H, Keys A, Simpson E, et al. The electrocardiogram in population studies. A classification system. Circulation. 1960; 21: 1160-1175, indexed in Pubmed: 13849070.

29. Alberti-Fidanza A, Fidanza F. Mediterranean Adequacy Index of Italian diets. Public Health Nutr. 2004; 7(7): 937-941, indexed in Pubmed: 15482621.

30. Menotti A, Kromhout D, Blackburn H, et al. Food intake patterns and 25-year mortality from coronary heart disease: cross-cultural correlations in the Seven Countries Study. The Seven Countries Study Research Group. Eur J Epidemiol. 1999; 15(6): 507-515, indexed in Pubmed: 10485342.

31. Pett KD, Kahn J, Willett WC, et al. White Paper. Ancel Keys and the Seven Countries Study. An evidence-based response to revisionist histories. Commissioned by The True Health Initiative. With emphasis on primary source material, historical records and review/critique by Seven Countries investigators, August 1. 2017: 1-64.

32. Keys A. Atherosclerosis: a problem in newer public health. J Mt Sinai Hosp N Y. 1953; 20(2): 118-139, indexed in Pubmed: 13085148

33. Yerushalmy J, Hilleboe HE. Fat in the diet and mortality from heart disease; a methodologic note. N Y State J Med. 1957; 57(14): 2343-2354, indexed in Pubmed: 13441073.

34. Sekikawa A, Miyamoto Y, Miura K, et al. Continuous decline in mortality from coronary heart disease in Japan despite a continuous and marked rise in total cholesterol: Japanese experience after the Seven Countries Study. Int J Epidemiol. 2015; 44(5): 1614-1624, doi: 10.1093/ije/dyv143, indexed in Pubmed: 26182938.

35. Piepoli MF, Hoes AW, Agewall S, et al. 2016 European guidelines on cardiovascular disease prevention in clinical practice: The Sixth Joint Task Force of the European Society of Cardiology and Other Societies on Cardiovascular Disease Prevention in Clinical Practice. Atherosclerosis. 2016; 252: 207-274.

36. Eckel RH, Jakicic JM, Ard JD, et al. 2013 AHA/ACC guideline on lifestyle management to reduce cardiovascular risk: a report of the American College of Cardiology/American Heart Association Task Force on Practice Guidelines. J Am Coll Cardiol. 2014; 63(25 Pt B): 2960-2984, doi:10.1016/j.jacc.2013.11.003, indexed in Pubmed: 24239922.

Cite this article as: Cybulska B, Kłosiewicz-Latoszek L. Landmark studies in coronary heart disease epidemiology. The Framingham Heart Study after 70 years and the Seven Countries Study after 60 years. Kardiol Pol. 2019; 77(2): 173-180, doi: 10.5603/KP.a2019.0017. 